\section{Linfoma de Burkitt en pacientes HIV positivo tratados con quimioterapia intensiva en el Hospital del Salvador entre 2011 y 2014. Protocolo Nacional de Cáncer del Adulto, Chile}

\author{
BÁRBARA PUGA L., KATHERINNE BUSTAMANTE K.a, \\ JAVIERA MOLINA E., ALEJANDRO ANDRADE M.
}

\section{Intensive chemotherapy for Burkitt lymphoma in HIV positive patients}

Background: Burkitt lymphoma has a low incidence, is highly aggressive, may be endemic, sporadic or associated with immunodeficiency and it has a high frequency of extranodal involvement. Overall and relapse free survival in HIV patients is 72 and $71 \%$ respectively. However, the current protocol in Chile considers a positive HIV serology as an exclusion criterion for intensive chemotherapy. Aim: To analyze the response to Burkitt lymphoma treatment among HIV positive patients. Material and Methods: All HIV positive patients with a Burkitt lymphoma treated using PANDA protocol in a public hospital were analyzed. Results: Eight male patients aged between 25 and 43 years, $63 \%$ in stage IV, were analyzed. All patients received an intensified chemotherapy regime, three of them without Rituximab. Complete remission was achieved in $87 \%$. One patient was refractory to treatment and one patient relapsed at 5 months and died. Overall and relapse free survival were 58 and $60 \%$ respectively. All patients had episodes of high risk febrile neutropenia, but it did not cause deaths. Conclusions: In this group of HIV patients, intensive chemotherapy for Burkitt lymphoma had a high degree of effectiveness with a low relapse rate and high cure rate.

(Rev Med Chile 2015; 143: 1505-1511)

Key words: Antineoplastic protocols; Burkitt lymphoma; HIV.

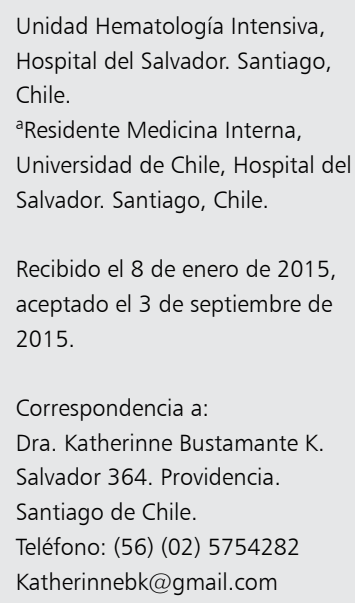

E 1 linfoma de Burkitt (LB) es un linfoma de células $B$ maduras altamente agresivo que corresponde a menos del $5 \%$ de los linfomas no-Hodgkin, con una incidencia en adultos de 2,5 casos por millón de habitantes por año ${ }^{1}$. En Chile, estudios recientes en el Hospital del Salvador (Santiago), evidenciaron que 21,8\% de los casos de linfoma no-Hodgkin estirpe B en pacientes con serología positiva para el virus de la inmunodeficiencia humana adquirida $(\mathrm{VIH}+)$ correspondieron a $\mathrm{LB}^{2}$. Otra serie de casos del Hospital San Borja Arriarán evidenció 47\% ${ }^{3}$.
El LB se caracteriza por la translocación y desregulación del oncogen c-MYC producto de la $t(8: 14)$, codificando para una proteína de transcripción que modula los procesos celulares de crecimiento, división, metabolismo y apoptosis ${ }^{4}$.

Existen otros factores asociados, como la infección por virus Ebstein Barr, que juegan un rol en los casos endémicos y en forma minoritaria en la asociación a inmunodeficiencia.

Existen tres formas clínicas; la endémica, que afecta a niños africanos entre 4 y 6 años y que se manifiesta con compromiso mandibular o tumor 
facial en $50-60 \%$ de los casos y que en menos de $10 \%$ hay compromiso de médula ósea ${ }^{5}$.

La forma esporádica se presenta en adultos menores de 35 años, con predominio en sexo masculino de $4: 1^{6}$, con compromiso abdominal y, frecuentemente, infiltración ósea (30\%), sistema nervioso central (SNC) (15\%) y también de renal, testicular, ovárico, mamario o médula ${ }^{7}$.

La tercera presentación ocurre en pacientes con inmunodeficiencia tales como VIH+, así como receptores de trasplante de órganos sólidos. En VIH, la afectación se presenta con recuentos de CD4 generalmente $>200$, por tanto, sin variaciones a pesar del advenimiento de la terapia antirretroviral ${ }^{8,9}$ y clínicamente suele presentarse con compromiso ganglionar, medular y SNC.

La presentación clínica se caracteriza por el crecimiento rápido de masas, presencia de síntomas $\mathrm{B}$ (fiebre, baja de peso y sudoración) y a menudo evidencia de lisis tumoral espontánea clínica.

Histológicamente, el LB presenta una infiltración monomórfica, con células de mediano tamaño, con núcleo redondeado y citoplasma basófilo, con vacuolas lipídicas citoplasmáticas prominentes. Tiene alta tasa de proliferación con Ki67+, 100\%, en todos los casos. Expresan IgM de superficie y antígenos asociados a la estirpe $B$ (CD19, 20, 22, 79a), así como CD10, HLA-DR y CD 43, ausencia de CD5, BCL-2, TdT y típicamente CD23.

La aproximación al tratamiento actual, adaptada de los esquemas pediátricos, consiste en ciclos de quimioterapia intensiva, con múltiples drogas asociadas, altas dosis de metotrexato y profilaxis del SNC. Los ciclos de quimioterapia se suceden en un lapso corto de tiempo (21 días, aproximadamente) por un período corto de 3 a 4 meses. El rituximab, un anticuerpo monoclonal anti CD20, asociado a las terapias actuales de quimioterapia intensiva ha logrado una mejoría en la remisión completa de $50 \%$ a $90 \%$, con sobrevida libre de enfermedad 4 años (SLE) de 74\% y sobrevida global (SG) de $78 \%{ }^{10}$. Los reportes en $\mathrm{VIH}+$ realizados en 2 cohortes evidenciaron $80 \%$ de remisión completa, SG a 4 años de $72 \%$ y SLE de $71 \%{ }^{11}$. Terapias de menor intensidad utilizadas en otros linfomas no-Hodgkin, como CHOP (ciclofosfamida, doxorubcina, vincristina y prednisona), demostraron tener una baja efectividad con alta tasa de recaída y baja tasa de curación ${ }^{12,13,14,15}$. El Protocolo Nacional de Burkitt excluye a los pacientes VIH+ de la posibilidad de quimioterapia intensiva.

El objetivo de este estudio fue caracterizar el $\mathrm{LB}$ en pacientes $\mathrm{VIH}+\mathrm{y}$ evaluar respuesta a tratamiento de los pacientes diagnosticados y tratados con el Protocolo Nacional de Burkitt con y sin rituximab, entre enero de 2011 y mayo de 2014 en la Unidad de Hematología Intensiva del Hospital del Salvador, Centro de Referencia del Servicio de Salud Metropolitano Oriente y de las regiones I, II, III y XV del país.

\section{Material y Métodos}

En el año 2011, en base a evidencia médica, el Comité Hemato-Oncológico de nuestro hospital decidió incorporar a los pacientes seropositivos para VIH con linfoma de Burkitt, en buenas condiciones generales, evaluando y dando a conocer los resultados, con el objeto de evaluar la pertinencia de este criterio de exclusión en el protocolo. El tratamiento, incluido el Rituximab ${ }^{\circledR}$, fueron aportados por nuestro hospital. Se analizaron todos los pacientes diagnosticados con linfoma de Burkitt y serología VIH+ diagnosticados entre enero de 2011 y mayo de 2014 que recibieron tratamiento intensificado según protocolo nacional PANDA con esquema Burkitt 2010 y su actualización 2013, previo consentimiento informado.

Fueron incluidos pacientes entre 15 y 60 años, con estado general compatible para recibir el tratamiento oncológico intensivo y certificación del diagnóstico a través de la fundamentación clínica, imagenológica, histológica e inmunohistoquímica del tumor. Fueron excluidos pacientes mayores de 60 años, aquellos con performance status incompatible para quimioterapia intensiva, trastorno psiquiátrico severo certificado por especialista, cáncer concomitante activo que comprometiera el pronóstico o cualquier otra patología que contraindicara la quimioterapia intensiva: insuficiencia renal crónica con creatinina $>2 \mathrm{mg} / \mathrm{dl}$, cirrosis hepática, insuficiencia cardiaca con fracción de eyección $<50 \%$, limitación crónica del flujo aéreo con insuficiencia respiratoria. La condición de $\mathrm{VIH}+$, al contrario de lo establecido en la guía, fue criterio exigido para este tratamiento piloto.

A todos los pacientes se les realizó exámenes preestablecidos según protocolo (Tabla 1 ). Los pacientes sin el diagnóstico previo de VIH se 
les realizó serología mediante ELISA y la confirmación fue realizada en el Instituto de Salud Pública y sometidos a prueba de identidad, previo consentimiento informado. La etapa clínica se estableció según el compromiso ganglionar y extraganglionar (Tabla 2) y el grupo de riesgo se estableció según la etapa clínica y los niveles de LDH (Tabla 3). El tratamiento intensificado fue realizado con el paciente hospitalizado. La preparación de la quimioterapia se llevó a cabo en una unidad centralizada bajo campana de flujo laminar y administrada según el grupo de riesgo. Los pacientes con SNC comprometido al diagnóstico recibieron adicionalmente quimioterapia intratecal triple, dos veces por semana, por al menos 8 administraciones y 2 últimos estudios citológicos de LCR negativos (Tabla 4).

La respuesta al tratamiento se realizó posterior al primer bloque AA (Tabla 4) con tomografía computarizada (TAC) de tórax, abdomen y pelvis, mielograma, en caso de compromiso de médula ósea (MO) al diagnóstico, citología de líquido céfalorraquídeo (LCR) en caso de compromiso SNC al diagnóstico.

Se estableció como remisión completa la ausencia de masa tumoral, de compromiso de $\mathrm{MO}$ o de infiltración de LCR, pudiendo alcanzarse posterior al primer o segundo ciclo de quimioterapia.

La remisión parcial fue definida como persistencia de masas residuales o blastos en LCR o MO de menor magnitud que al diagnóstico posterior al segundo ciclo de quimioterapia ( $\left.1^{\text {er }} \mathrm{BB}\right)$. Los pacientes en esta condición recibieron como $3^{\text {er }} \mathrm{y}$ $6^{\circ}$ ciclo de quimioterapia un bloque CCR.

La refractariedad fue definida como persistencia de enfermedad tumoral posterior a primer ciclo CC, caso en el cual el paciente era candidato a cuidados paliativos.

La terapia antirretroviral se inició en los casos nuevos y se mantuvo en los pacientes previamente diagnosticados, siguiendo los esquemas establecidos por el Comité de Infectología del Hospital del Salvador. No se procedió a realización de carga viral ni recuento de CD4, dado permanentes períodos de aplasia severa y linfopenia absoluta secundaria a quimioterapia.

\section{Resultados}

Entre enero de 2011 y mayo de 2014, 13 pacientes fueron diagnosticados con linfoma de Burkitt.
De ellos, 8 (62\%) resultaron con serología VIH positiva. Todos recibieron quimioterapia intensiva según Protocolo Nacional de Burkitt y fueron incluidos en el análisis.

Las características clínicas de los 8 pacientes con LB y HIV+ se muestran en la Tabla 5, con un rango etario entre 25 y 47 años (mediana de 31,5 años), todos de sexo masculino. En 5 pacientes su condición VIH+ era conocida previamente, mientras en 3 casos el diagnóstico se estableció en el estudio inicial establecido por el protocolo.

Seis pacientes presentaban comorbilidades previas, como abuso de sustancias ilegales en 3, alcoholismo en 1, serología virus hepatitis B en 2, hipertensión arterial y diabetes mellitus no insulino requirente en 1 .

La presentación clínica al diagnóstico con síntomas B fue descrita en 50\% de los pacientes. El compromiso extraganglionar fue evidenciado en 5 pacientes, 2 pacientes presentaron compromiso de médula ósea, sin evidencia clínica de falla medular. Dos pacientes tenían compromiso de SNC, uno de ellos con amaurosis de ojo izquierdo y parálisis facial periférica, el otro paciente presentó células

\section{Tabla 1. Evaluación inicial en Linfoma de Burkitt}

- Hemograma con fórmula leucocitaria y recuento de plaquetas

- Tiempo de protrombina (TP), tiempo de tromboplastina (TTPK)

- Perfil bioquímico con estudio de función renal y hepática

- Deshidrogenada láctica sérica (LDH)

- TAC Tórax, abdomen y pelvis

- Ecocardiograma doppler a todo paciente > 50 años o con factores de riesgo

- Estudio de marcadores virales (VHB, VHC, HIV, VDRL, HTVL-I, Chagas)

- Mielograma y/o biopsia de médula ósea (MO)

- Inmunofenotipo por citometría de flujo

- Cariograma (estudio citogenético) (si la MO está comprometida)

- Estudio de líquido cefalorraquídeo (LCR)

* Lista de exámenes según el Protocolo Cáncer de adultos, Ministerio de Salud 2013-2014 (PANDA), sección Protocolo de tratamientos Linfoma de células pequeñas no hendidas o Linfoma de Burkitt y Leucemia Linfoblástica B Madura. Encargadas Dras. María Elena Cabrera y Bárbara Puga. 
neoplásicas en LCR. Un paciente presentó lisis tumoral espontánea, con falla renal que corrigió con medidas médicas, sin necesidad de terapia de reemplazo. Siete pacientes se encontraban en riesgo 3 y 5 pacientes, concomitantemente, en etapa IV (Tabla 6).

Tres pacientes fueron diagnosticados durante el año 2011 y recibieron un esquema intensificado de quimioterapia sin rituximab, el que fue incorporado a partir del año 2012. A partir de ese año, 5 pacientes recibieron quimioterapia intensiva asociada a rituximab.

\section{Tabla 2. Definición de etapa clínica}

\begin{tabular}{|ll|}
\hline Etapa I & $\begin{array}{l}\text { Compromiso nodal o extraganglionar único, } \\
\text { excluido el mediastino y abdomen }\end{array}$ \\
Etapa II & $\begin{array}{l}\text { Dos o más áreas ganglionares a un lado del } \\
\text { diafragma o tumor abdominal resecable }\end{array}$ \\
Etapa III & $\begin{array}{l}\text { Tumor a ambos lados del diafragma } \\
\text { Tumor intratoráxico } \\
\text { Compromiso abdominal extenso } \\
\text { Compromiso extradural }\end{array}$ \\
Etapa IV & $\begin{array}{l}\text { Compromiso inicial de médula ósea, SNC o } \\
\text { esquelético multifocal }\end{array}$ \\
\hline
\end{tabular}

*Tabla clasificación Ann Arbor. Protocolo Cáncer de adultos, Ministerio de Salud 2013-2014 (PANDA), sección Protocolo de tratamientos Linfoma de células pequeñas no hendidas o Linfoma de Burkitt y Leucemia Linfoblástica B Madura. Encargadas Dras. María Elena Cabrera y Bárbara Puga.
Todos los pacientes recibieron profilaxis con cotrimoxazol diario, itraconazol en dosis altas, aciclovir, levofloxacino, además de factor estimulante de colonias granulocíticas, en cada período de aplasia. Posterior a la administración de $1^{\circ}$ bloque de quimioterapia y durante el período de aplasia, todos los pacientes cursaron con neutropenia febril de alto riesgo (NFAR) con múltiples focos

\section{Tabla 3. Clasificación de riesgo en Linfoma de Burkitt}

$\begin{array}{ll}\text { Riesgo } 1 & \begin{array}{l}\text { Estadíos I y II } \\ \text { Tumor primario resecado completamente en } \\ \text { forma macroscópica }\end{array} \\ \text { Riesgo } 2 & \begin{array}{l}\text { Estadío III con } \mathrm{LDH}<500 \mathrm{u} / \mathrm{l} \text {, medido antes } \\ \text { de iniciada la quimioterapia } \\ \text { Tumor primario resecado en forma incom- } \\ \text { pleta }\end{array} \\ \text { Riesgo } 3 \quad & \text { Estadío III con LDH }>500 \mathrm{~m} / \mathrm{I} \\ & \text { Estadío IV } \\ & - \text { Compromiso de SNC } \\ & - \text { Compromiso de médula ósea } \\ & - \text { Compromiso esquelético multifocal } \\ & - \text { Leucemia B (> 25\% linfocitos tipo L3 en } \\ & \text { médula ósea) }\end{array}$

*Protocolo Cáncer de adultos, Ministerio de Salud 20132014 (PANDA), sección Protocolo de tratamientos Linfoma de células pequeñas no hendidas o Linfoma de Burkitt y Leucemia Linfoblástica B Madura. Encargadas Dras. María Elena Cabrera y Bárbara Puga.

Tabla 4. Esquema de tratamiento según grupo de riesgo

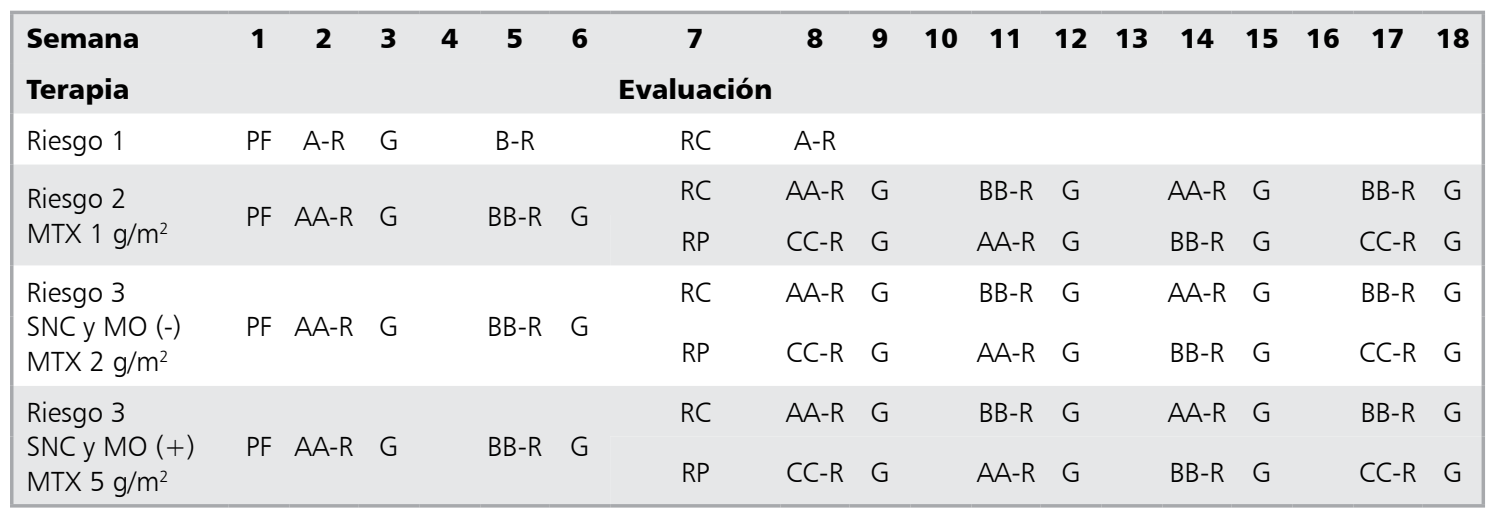

*Obtenido de Protocolo Cáncer de adultos, Ministerio de Salud 2013-2014 (PANDA), sección Protocolo de tratamientos Linfoma de células pequeñas no hendidas o Linfoma de Burkitt y Leucemia Linfoblástica B Madura. Encargadas Dras. María Elena Cabrera y Bárbara Puga. SNC: Compromiso de sistema nervioso central. MO: Compromiso de médula ósea. MTX: Metotrexato. G: G-CSF (factor estimulante de colonias granulocítica). PF: Pre fase. A-R: Block A más Rituximab®. B-R: Block B más Rituximab®. AA-R: Block AA más Rituximab ${ }^{\circledR}$. BB-R: Block BB más Rituximab ${ }^{\circledR}$. CC-R: Block CC más Rituximab ${ }^{\circledR}$ 
Tabla 5. Característica de los pacientes al diagnóstico

\begin{tabular}{|lcc|}
\hline & n & (\%) \\
\hline Edad & $25-47$ Mediana \\
& 31,5 \\
\hline Sexo & & \\
\hline Femenino & & \\
Masculino & 0 & \\
VIH positivo & 8 & $(100)$ \\
Previamente conocido & 8 & \\
Diagnóstico simultáneo & 5 & $(63)$ \\
TARV & 3 & $(37)$ \\
\hline
\end{tabular}

Tabla 6. Características del Linfoma de Burkitt al diagnóstico

\begin{tabular}{|lll|}
\hline & n & (\%) \\
Compromiso & & \\
Síntomas B & 4 & $(50)$ \\
Compromiso extra ganglionar & 5 & $(63)$ \\
LCR (+) & 2 & $(25)$ \\
MO (+) & 2 & $(25)$ \\
Riesgo & & \\
1 & 1 & $(13)$ \\
2 & 0 & \\
3 & 7 & $(88)$ \\
Estadío & & \\
I & 2 & $(25)$ \\
II & 0 & \\
III & 1 & $(13)$ \\
IV & 5 & $(63)$ \\
\hline
\end{tabular}

involucrados. El compromiso pulmonar se presentó en 6 pacientes. En uno de ellos, la etiología sospechada fue Pneumocytis jiroveci y en otro aspergilosis pulmonar invasiva. En ninguno de ellos se pudo confirmar patógeno. Dada la gravedad del cuadro, se evitó la realización de procedimientos invasivos y recibieron tratamiento empírico con resolución del cuadro. El foco digestivo también fue frecuente, evidenciándose en 6 pacientes. En 1 paciente con cuadro de diarrea se confirmó toxina para Clostridium difficili. Un foco perianal se presentó en 3 pacientes, foco cutáneo, asociado a vías venosas, en 3 pacientes y en 2 pacientes se describió foco oral. Cinco pacientes presentaron asociación de más de un foco. Los episodios de
NFAR fueron entre 1 a 4 en cada paciente, con un promedio de 2 .

Todos los episodios de NFAR fueron abordados según el protocolo nacional, con esquemas antibióticos de excepción escalonados (ceftriaxona, tazonam, imipenem, asociados a amikacina y vancomicina, colistin, tigeciclina y linezolid en situaciones puntuales. El uso del antifúngico anfotericina $B$ fue indicado en 3 pacientes en contexto de fiebre persistente o sospecha de aspergillosis pulmonar invasiva por TAC. Un paciente requirió traslado a UCI, con requerimientos de drogas vasoactivas y ventilación mecánica no invasiva, siendo trasladado a sala a las $48 \mathrm{~h}$.

Otras reacciones adversas observadas durante el período de tratamiento fueron: síndrome convulsivo en 1 paciente, sin causa estructural asociada y que se planteó en contexto farmacológico, hepatotoxicidad farmacológica recuperada en 1 paciente y cardiotoxicidad que se manifestó como bradicardia sin necesidad de apoyo con drogas vasoactivas ni marcapaso, en probable contexto séptico y farmacológico, en 1 paciente.

Con respecto a la respuesta a tratamiento, se evidenció RC en 5 de 8 (62\%) pacientes posterior al primer ciclo de quimioterapia, en 6 de 8 (75\%), después del segundo ciclo de quimioterapia y en 7 de $8(87 \%)$ posterior al tercer ciclo. Un paciente fue refractario a tratamiento, siendo incorporado a la Unidad de Cuidados Paliativos y Alivio del Dolor. No se observó mortalidad asociada a tratamiento. De los 7 pacientes que alcanzaron RC, 1 falleció en accidente de tránsito, 1 paciente que recibió quimioterapia sin rituximab, recayó a los 5 meses, siendo ingresado a la Unidad de Cuidados Paliativos. Cinco pacientes se mantienen en remisión completa con una sobrevida global y libre de recaída a 4 años de $58 \%$ y $60 \%$ respectivamente (Figuras 1 y 2 ).

\section{Discusión}

En este estudio que agrupó pacientes con LB y serología VIH positiva, sólo se reportaron casos menores de 50 años (mediana 31,5, rango 25 y 43 años), todos de sexo masculino.

A diferencia de lo referido por otros trabajos realizados en Chile, donde aproximadamente en la mitad de los casos la condición de HIV se hizo evidente sólo al momento del diagnóstico ${ }^{2}$, en este 

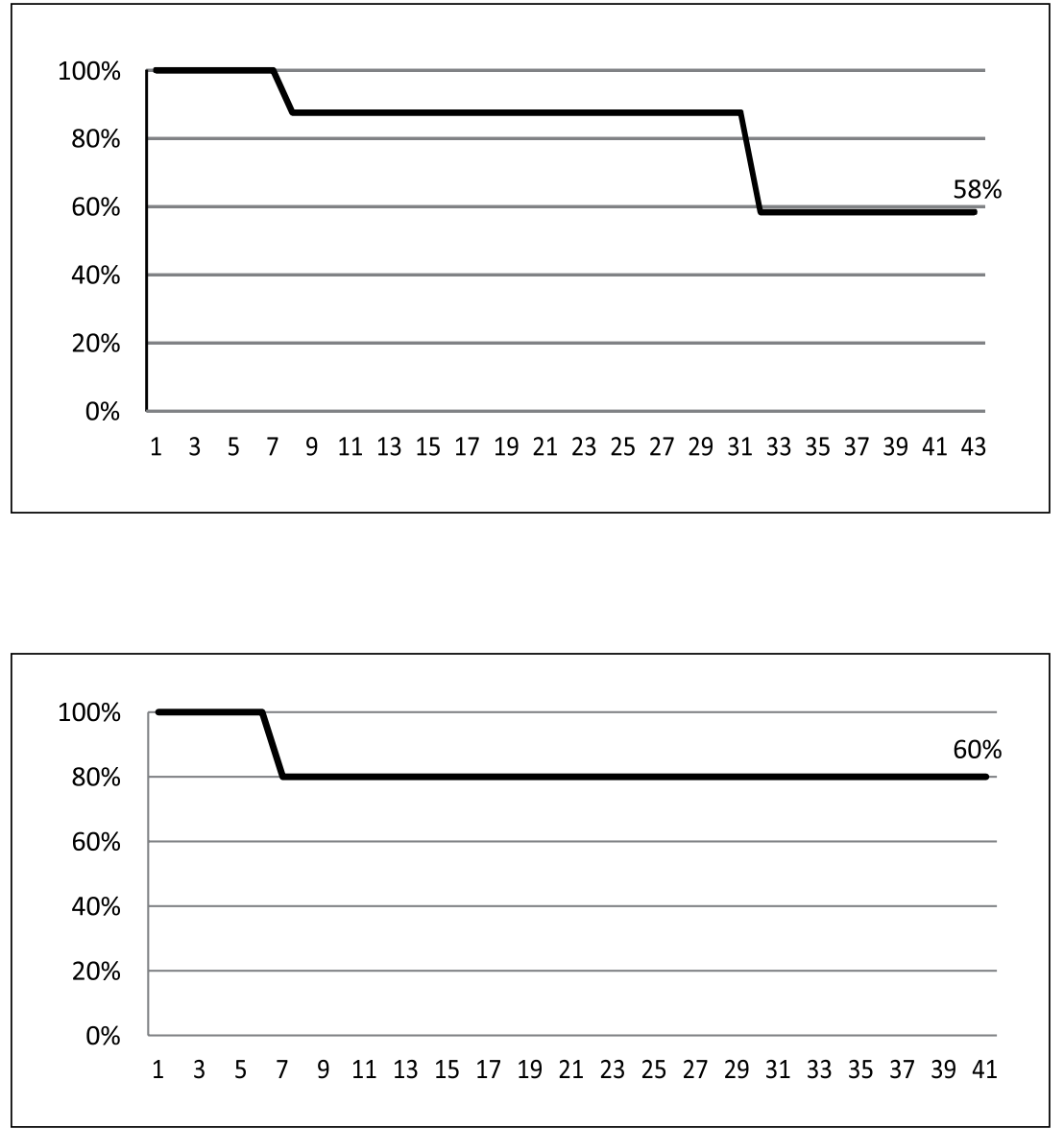

Figura 1. Linfoma de Burkitt en HIV $(+)$. Sobrevida global; $\mathrm{n}=8$.
Figura 2. Linfoma de Burkitt en $\operatorname{HIV}(+)$. Sobrevida Libre de Recaída; $\mathrm{n}=7$. estudio, en la mayoría de los pacientes $(62,5 \%)$ su condición serológica ya era conocida al momento del diagnóstico de LB y sólo 37,5\% fue conocida en forma concomitante. Esta cifra es similar a estudios realizados en el Reino Unido ${ }^{16}$, evidenciando el cambio favorable hacia la pesquisa y acceso de la población a la toma de exámenes. Las manifestaciones clínicas son similares a lo reportado en la literatura, con compromiso extranodal habitual, siendo el abdominal el más frecuente ${ }^{17,18}$. La mayoría de los pacientes (75\%) se presentaron con linfoma en estadíos avanzados (III-IV), similar a lo obtenido en otros trabajos ${ }^{19}$. El compromiso de MO se observó en 25\%, similar a otros estudios que lo reportan en $22-38 \%$. En nuestro estudio el compromiso de SNC fue encontrado en $25 \%$, más frecuente que el 12-17\% descrito en la literatura $^{7,20}$. La toxicidad relacionada a quimiotera- pia intensiva asociada a rituximab en pacientes HIV+ evidencia mayor presencia de mucositis e infecciones severas, sin embargo, no se describen diferencias significativas en la mortalidad asociada a tratamiento ${ }^{21}$. En este estudio, aunque cada bloque de quimioterapia se asoció a NFAR, no hubo mortalidad relacionada.

El uso de quimioterapia intensiva en pacientes con LB asociado a rituximab ha logrado RC hasta $90 \%$ de los pacientes, con una SG 78\% y SLE $71 \%{ }^{10}$. En pacientes VIH se han descrito cifras similares con RC cercanas a $80 \%$, SG 72\% y SLE $71 \%{ }^{11}$. En nuestro estudio se evidenció una RC $87,5 \%$ con SG y SLE a 4 años de $58 \%$ y $60 \%$, apoyando la necesidad de incluir en los protocolos nacionales de quimioterapia intensiva para LB a pacientes VIH, actualmente considerados como criterio de exclusión. 


\section{Referencias}

1. Perkins A, Friedber JW. Burkitt Lymphoma in Adults. Hematology Am Soc Hematol Educ Program 2008: 3418.

2. Cabrera ME, Silva G, Soto A, Roselló R. Castro C, Martínez $\mathrm{V}$, et al. [HIV-related lymphoma in a public hospital in Chile. Analysis of 55 cases]. Rev Med Chile 2012; 140 (2): 243-50.

3. Pizarro A, García H, Riquelme A, Carmona J, Cortés C. [Lymphomas associated with HIV infection in patients at the Hospital San Borja Arriarán/Fundación Arriarán 2001-2008, Santiago, Chile]. Rev Chilena Infectol 2013; 30 (1): 23-30.

4. Hecht JL, Aster JC. Molecular biology of Burkitt's lymphoma. J Clin Oncol 2000; 18: 3707.

5. Magrath I. Epidemiology: clues to the pathogenesis of Burkitt lymphoma. Br J Haematol 2012; 156: 744.

6. Sant M, Allemani C, Tereanu C, De Angelis R, Capocaccia $\mathrm{R}$, Visser $\mathrm{O}$, et al. and the HAEMACARE Working Group. Incidence of hematologic malignancies in Europe by morphologic subtype: results of the HAEMACARE project. Blood 2010; 116: 3724-34.

7. Blum KA, Lozanski G, Byrd JC. Adult Burkitt leukemia and lymphoma. Blood 2004; 104: 10.

8. Costa L, Xavier A, Wahlquist A, Hill E. Trends in survival of patients with Burkitt lymphoma/leukemia in the USA: an analysis of 3691 cases. Blood 2013; 121 (24): 4861-6.

9. Guech-Ongey M, Simard EP, Anderson WF, Engels EA, Bhatia K, Devesa SS, et al. AIDS-related Burkitt lymphoma in the United States: what do age and CD4 lymphocyte patterns tell us about etiology and/or biology? Blood 2010; 116: 5600-4.

10. Kieron Dunleavy. How I treat HIV-associated lymphoma. Blood 2012; 119: 3245-55.

11. Rizzieri DA, Johnson JL, Byrd JC, Lozanski G, Blum KA, Powell B, et al. and the Alliance for Clinical Trials In Oncology (ACTION) (2014), Improved efficacy using rituximab and brief duration, high intensity chemotherapy with filgrastim support for Burkitt or aggressive lymphomas: cancer and Leukemia Group B study 10 002. Br J Hematol 165: 102-11.

12. Xicoy B, Ribera JM, Müller M, García O, Hoffmann $\mathrm{C}$, Oriol A, et al. Dose-intensive chemotherapy including rituximab is highly effective but toxic in human immunodeficiency virus-infected patients with Burkitt lymphoma/leukemia: parallel study of 81 patients. Leuk Lymphoma. 2014; 55: 2341-8.

13. Dunleavy K, Pittaluga S, Shovlin M, Steinberg S, Cole D, Cliona Grant C, et al. Low-Intensity Therapy in Adults with Burkitt's Lymphoma. N Engl J Med 2013; 369: 1915-25.

14. Thomas DA, Faderl S, O’Brien S, Bueso-Ramos C, Cortés J, García-Manero G, et al. Chemoimmunotherapy with hyper-CVAD plus rituximab for the treatment of adult Burkitt and Burkitt-type lymphoma or acute lymphoblastic leukemia. Cancer 2006; 106: 1569-80.

15. Wästerlid T, Brown $P$, Hagberg $O$, Hagberg H, Pedersen L, D'Amore F, et al. Impact of chemotherapy regimen and rituximab in adult Burkitt lymphoma: a retrospective population-based study from the Nordic Lymphoma Group. Ann Oncol 2013; 24: 1879-86.

16. Bower M, Fisher M, Hill T, Reeves I, Walsh J, Orkin $\mathrm{C}$, et al. CD4 counts and the risk of systemic nonHodgkin's lymphoma in individuals with HIV in the UK. Haematologica 2009; 94: 875-80.

17. Mead G, Sydes M, Walewski J, Grigg J, Hatton C, Norbert $\mathrm{P}$, et al. An international evaluation of CODOX-M and CODOX-M alternating with IVAC in adult Burkitt's lymphoma: results of United Kingdom Lymphoma Group LY06 study. Ann Oncol 2002;13: 1264-74.

18. Soussain C, Ostronoff P, Delmer A, Rigal-Huguet F, Cambier N, Leprise PY, et al. Small noncleaved cell lymphoma and leukemia in adults. A retrospective study of 65 adults treated with the LMB pediatric protocols. Blood 1995; 85: 664-74.

19. Diebold J, Raphael M, Prévot S, Audouin J. Lymphomas associated with HIV infection. Cancer Surv 1997; 30: 263-93.

20. Cairo MS, Sposto R, Perkins SL, Meadows AT, Hoover-Regan ML, Anderson JR, et al. Burkitt's and Burkitt-like lymphoma in children and adolescents: a review of the Children's Cancer Group experience. $\mathrm{Br}$ J Haematol 2003; 120: 660-70.

21. Oriol A, Ribera JM, Bergua J, Giménez Mesa E, Grande C, Esteve J, et al. High dose chemotherapy in adult Burkitt lymphoma: Comparison of results in Human Immunodeficiency Virus-infected and non-infected patients. Cancer 2008; 113: 117-25. 